\title{
Time-Domain Channel Estimation Scheme for OFDM over Fast Fading Channels
}

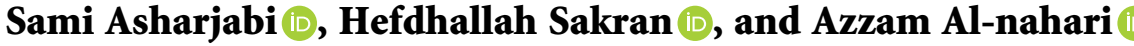 \\ Department of Electrical Engineering, Faculty of Engineering, Ibb University, Ibb, Yemen \\ Correspondence should be addressed to Sami Asharjabi; sami.tarbosh@gmail.com
}

Received 5 January 2022; Accepted 5 February 2022; Published 27 February 2022

Academic Editor: Hamada Esmaiel

Copyright ( $(2022$ Sami Asharjabi et al. This is an open access article distributed under the Creative Commons Attribution License, which permits unrestricted use, distribution, and reproduction in any medium, provided the original work is properly cited.

\begin{abstract}
In high-mobility scenarios, the time variation of mobile radio channels leads to a loss of orthogonality among subcarriers in orthogonal frequency division multiplexing (OFDM) systems, resulting in intercarrier interference (ICI) and performance deterioration. Conventional channel estimation schemes are usually based on pilot tones, which are distributed in each OFDM symbol to estimate the channel variation. Hence, the channel estimator itself suffers from ICI. In this study, a new estimation scheme, which does not suffer from ICI, is proposed to estimate the channel variation within OFDM symbols. The main idea is to zero-pad (ZP) the OFDM symbol in the time domain. Then, in the middle of the ZP interval, an impulse signal is inserted as a pilot sample, which is used to estimate the channel at the pilot signal in the OFDM symbol. Finally, a linear model is used to estimate the channel variation over an OFDM symbol. Additionally, we derive the mean squared error (MSE) of the proposed estimation technique under the constraint that the channel varies linearly within OFDM symbols. Simulation results show that our scheme can achieve a substantial improvement in the bit error rate (BER) performance of OFDM, in spite of the OFDM symbol length being increased. Moreover, in many cases, the new scheme can achieve the same BER performance as the perfect knowledge of channel state information (CSI). Theoretical analysis and numerical simulations show that our scheme achieves excellent performance with much lower computational complexity.
\end{abstract}

\section{Introduction}

Due to the rapid deployment of high-speed vehicles, such as high-speed railway and low-altitude fly objects systems, during the past few years, wireless communication systems should be able to provide reliable service to the mobile devices in such high-mobility environment $[1,2]$. Thus, high-mobility communications have become an integral part of the fifth generation (5G) of wireless systems standards, which first deployed in the year 2020 [3, 4]. The $5 \mathrm{G}$ communication systems are expected to support high speed up to $500 \mathrm{~km} / \mathrm{h}$ and provide high data rate up to $150 \mathrm{Mb} / \mathrm{s}$, simultaneously [5].

To achieve the $5 \mathrm{G}$ requirements, many technologies have been proposed, such as massive multiple-input multipleoutput (MIMO) systems. Orthogonal frequency division multiplexing (OFDM) combined with massive MIMO is a promising technique for wideband massive MIMO transmission [6]. OFDM is one of the most attractive modulation techniques due to its high spectral efficiency and its robustness against multipath delay. Recently, index modulation-OFDM-spread spectrum (IM-OFDM-SS) [7] and low-redundant energy UW-OFDM (LRE-UW-OFDM) [8] schemes have been proposed to improve the spectral and energy efficiencies, respectively, in OFDM systems. OFDM has been extensively used in wired and wireless application, digital audio/video broadcast (DAB/DVB), and many standards such as IEEE 802.16a and IEEE 802.11e $[9,10]$.

However, OFDM is vulnerable to the time variation of the channel, which is one of its main drawbacks. In highmobility environments, the time variation of the channel destroys the orthogonality of the subcarriers severely, resulting in intercarrier interference (ICI) and performance degradation $[11,12]$. Usually, the normalized maximum Doppler frequency, $\varepsilon=f_{d, \max } T_{u}$, is used to measure the time variation of the channel, where $f_{d, \max }$ is the maximum 
Doppler spread, $T_{u}=1 / \Delta f$ is the useful OFDM symbol duration, and $\Delta f$ is the subcarriers spacing.

\section{Related Work}

To estimate the channel variation over several OFDM symbols or within one OFDM symbol, different estimation techniques have been proposed. Most channel estimation techniques are based on pilots' (arrangement in OFDM) symbols $[13,14]$. In fast time-varying channel environments, the channel variation during one OFDM symbol period exhibits high-order variation $[15,16]$. Hence, the number of unknown channel coefficients to be estimated is very large. To reduce the number of estimated coefficients, various models have been developed to approximate the channel variation, such as a basis expansion model (BEM) $[17,18]$, a block-sparse Bayesian learning (BSBL) $[19,20]$, and a piecewise linear model (PLM) [21].

In this paper, a zero-padding (ZP) scheme is proposed to estimate the channel variation in OFDM systems, which can be summarized as follows. First, a cyclic prefixed OFDM symbol is zero-padded. After that, an impulse signal is inserted in the middle of these zeros, which is used in the receiver to estimate the channel at the pilot sample of each OFDM symbol. Lastly, a linear model is used to estimate the channel variation within the OFDM symbol period.

The remainder of this paper is organized as follows. First, the proposed model of an OFDM system is described briefly in Section 3. Then, the ICI due to time-varying channel is described in Section 4. The channel estimation is presented in Section 5. Simulation results are presented and discussed in Section 6. Finally, in Section 7, the conclusions of this paper are drawn.

Notations: superscripts $(.)^{-1},(.)^{H}$, and $(.)^{T}$ stand for inverse, conjugate transpose (Hermitian), and transpose operators, respectively. $[\mathbf{A}]_{P}, \operatorname{diag}(),. \mathbf{J}_{M \times N}$, and $\mathbf{I}_{Q}$ denote a submatrix of $\mathbf{A}$ with row indices which correspond to the set $P$, a diagonal matrix is constructed from the vector-valued argument, an $M \times N$ is all-ones matrix, and $Q \times Q$ is identity matrix, respectively. $\|\cdot\|_{F}$ denotes the Frobenius matrix norm. The notation $\mathbb{C}^{M \times N}$ represents the set of $M \times N$ matrices in the complex field.

\section{System Model}

Figure 1 shows a discrete model of a baseband OFDM system with the proposed scheme (dashed line blocks). The OFDM system with the proposed scheme is different from conventional OFDM systems by adding and removing ZP blocks, after and before adding and removing a cyclic prefix (CP), respectively. After IDFT is performed, the $i$ th timedomain OFDM signal can be expressed as

$$
x_{n}^{(i)}=\frac{1}{\sqrt{N}} \sum_{k=0}^{N-1} X_{k}^{(i)} e^{j 2 \pi k n / N}, \quad 0 \leq n \leq N-1,
$$

where $N$ is the number of subcarriers and $X_{k}^{(i)}$ is the data symbol transmitted on the $k$ th subcarrier.

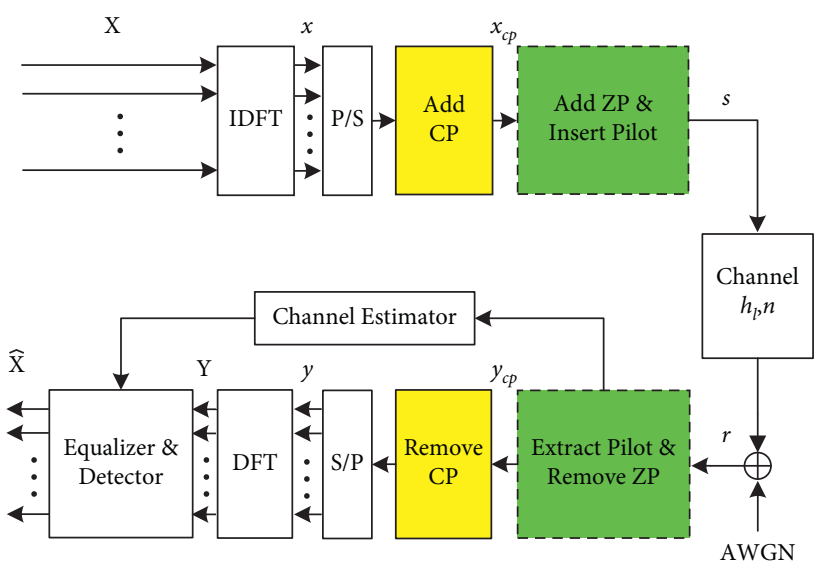

FIgURE 1: A baseband model of an OFDM system with the proposed scheme.

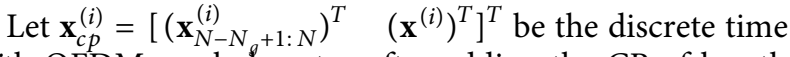
of $i$ th OFDM symbol vector after adding the CP of length $N_{g}$, that is, longer than the maximum delay spread of the channel impulse responses (CIR), where $\mathbf{x}^{(i)}=\left[x_{0}^{(i)}, x_{1}^{(i)}, \ldots, x_{N-1}^{(i)}\right]^{T}$. In our scheme, first, the cyclic prefixed symbol is extended by an interval (of zero samples) of total length $2 N_{g}+1$. Then, an impulse signal, $A \delta_{-\left(2 N_{g}+1\right)}$, with amplitude $A$, is inserted in the middle of these zeros, which is served as a pilot. The guard interval on the left of the impulse signal is used for eliminating intersymbol interference (ISI) caused by multipath fading channels, whereas the guard interval on the right is used for channel estimation. Hence, the OFDM symbol with the time-domain pilot samples can be written as follows:

$$
\mathbf{s}^{(i)}=[\underbrace{0, \ldots, 0, A \delta_{N / 2}, 0, \ldots, 0}_{2 N_{g}+1},\left(\mathbf{x}_{c p}^{(i)}\right)^{T}]^{T},
$$

where $\delta(\cdot)$ denotes the Kronecker delta function.

\section{ICI Analysis}

Assuming perfect synchronization of time, the received samples of the $i$ th OFDM symbol, after transmitting over a time-varying multipath channel, can be expressed as (after discarding both $\mathrm{ZP}$ and $\mathrm{CP}$ samples)

$$
y_{n}^{(i)}=\sum_{l=0}^{L-1} h_{l, n}^{(i)} x_{n-l}^{(i)}+w_{n}^{(i)}, \quad 0 \leq n \leq N-1,
$$

where $h_{l, n}^{(i)}$ is the gain of $l$ th time-domain channel path at the $n$th sample, $L$ is the total number of propagation paths, and $w_{n}^{(i)}$ is the additive white Gaussian noise (AWGN). Let us define the DFT of $Y_{k}^{(i)}$ as

$$
Y_{k}^{(i)}=\frac{1}{\sqrt{N}} \sum_{n=0}^{N-1} y_{n}^{(i)} e^{-j 2 \pi k n / N}, \quad 0 \leq k \leq N-1 .
$$

Substituting (3) into (4) and with some manipulations, the DFT of $y_{n}^{(i)}$ and $Y_{k}^{(i)}$ can be written as 


$$
Y_{k}^{(i)}=H_{k, k}^{(i)} X_{k}^{(i)}+\underbrace{\sum_{\substack{m=0 \\ m \neq k}}^{N-1} H_{k, m}^{(i)} X_{m}^{(i)}}_{\text {ICI term }}+W_{k}^{(i)}, \quad 0 \leq k \leq N-1,
$$

where

$$
\begin{aligned}
W_{k}^{(i)} & =\frac{1}{\sqrt{N}} \sum_{n=0}^{N-1} w_{n}^{(i)} e^{-j 2 \pi k n / N}, \quad 0 \leq k \leq N-1, \\
H_{k, m}^{(i)} & =\frac{1}{N} \sum_{n=0}^{N-1} \sum_{l=0}^{L-1} h_{n, l}^{(i)} e^{j 2 \pi n(m-k) / N} e^{j 2 \pi m l / N},
\end{aligned}
$$

where $H_{k, m}^{(i)}$ is a channel coefficient, which represents the ICI interference, due to Doppler spread, from the $m$ th subcarrier on $k$ th subcarrier. Equation (5) can be written in the matrix form as follows:

$$
\mathbf{Y}^{(i)}=\mathbf{H}^{(i)} \mathbf{X}^{(i)}+\mathbf{W}^{(i)}
$$

where $\quad \mathbf{X}^{(i)}=\left[X_{0}^{(i)}, X_{1}^{(i)}, \ldots, X_{N-1}^{(i)}\right]^{T}, \quad \mathbf{Y}^{(i)}=\left[Y_{0}^{(i)}, Y_{1}^{(i)}\right.$, $\left.\ldots, Y_{N-1}^{(i)}\right]^{T}, \mathbf{W}^{(i)}=\left[W_{0}^{(i)}, W_{1}^{(i)}, \ldots, W_{N-1}^{(i)}\right]^{T}$, and $\mathbf{H}^{(i)}$ is the frequency-domain channel matrix, given by (for simplicity, the OFDM symbol index $i$ is omitted)

$$
\mathbf{H}=\left[\begin{array}{ccccc}
H_{0,0} & H_{0,1} & \ldots & H_{0, N-1} & \\
H_{1,0} & H_{1,1} & \ldots & H_{1, N-1} & \\
\vdots & \vdots & \vdots & \ddots & \vdots \\
H_{N-1,0} & H_{N-1,1} & \ldots & H_{N-1, N-1} &
\end{array}\right] .
$$

\section{Channel Estimation}

One should note that, in our proposed scheme, the received samples at $n_{l}=-2 N_{g}-1+l(0 \leq l \leq L-1)$ comprise the CIR corresponding to the pilot sample in an OFDM symbol. Hence, the channel path's variation during one OFDM symbol can be estimated by using a linear model, as depicted in Figure 2.

Let $\mathbf{r}^{(i)}$ denote the $i$ th received vector, where $\mathbf{r}^{(i)}=\left[r_{-\left(3 N_{g}+1\right)}^{(i)}, r_{-3 N_{g}}^{(i)} \ldots, r_{-1}^{(i)}, r_{0}^{(i)}, r_{1}^{(i)}, \ldots, r_{N-1}^{(i)}\right]^{T} \in \mathbb{C}^{N_{T} \times 1}$, where $N_{T}=N+3 N_{g}^{g}+1$.

The CIR through $\mathbf{r}^{(i)}$ can be extracted by using row selector matrix $\Psi=\left[\mathbf{I}_{N_{T}}\right]_{\mathscr{P}} \in \mathbb{C}^{L \times N_{T}}$, while $\mathscr{P}=$ $\left\{n_{0}, n_{1}, \ldots, n_{L-1}\right\}$ denotes the set of row indices corresponding to the time-domain pilot samples locations.

For symbol $\mathbf{r}^{(i)}$, the CIR can be written as

$$
\left[\mathbf{r}^{(i)}\right]_{\mathscr{P}}=\Psi \mathbf{r}^{(i)} \text {. }
$$

Then, the channel path gains at the pilot samples are obtained by using the conventional least-squares (LS) method. Let $\widehat{h}_{l, n_{l}}^{(i)}$ and $\widehat{h}_{l, n_{l}}^{(i+1)}$ denote, respectively, the LS estimated CIR corresponding to the pilot sample of the current $\mathbf{s}^{(i)}$ and the next $\mathbf{s}^{(i+1)}$ symbols of the $l$ th path at $n_{l}$. Then, the time-domain variation of the lth path $\widehat{\mathbf{h}}_{l}^{(i)}=\left[\widehat{h}_{l, 0}^{(i)}\right.$, $\left.\widehat{h}_{l, 1}^{(i)}, \ldots, \widehat{h}_{l, N-1}^{(i)}\right]^{T}$ in the current OFDM symbol can be estimated as follows:

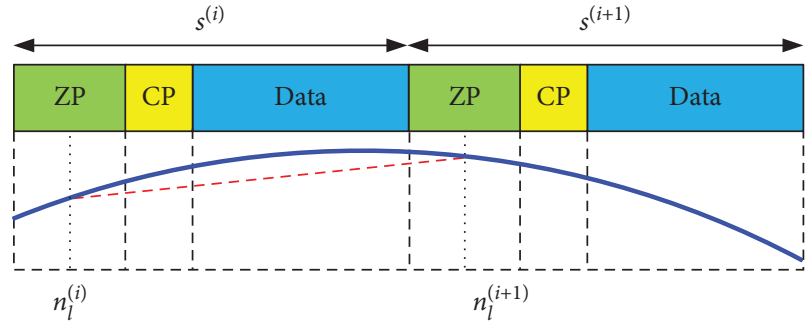

Figure 2: Channel approximation. Solid line: real or imaginary part of a channel path. Dashed line: linear model approximation of the channel path.

$$
\widehat{h}_{l, n}^{(i)}=\frac{\left(\widehat{h}_{l, n_{l}}^{(i+1)}-\widehat{h}_{l, n_{l}}^{(i)}\right)\left(n-n_{l}^{(i)}\right)}{N_{T}-1}+\widehat{h}_{l, n_{l}}^{(i)}
$$

where $n=0,1, \ldots, N-1, i=1,2, \ldots, l=0,1, \ldots, L-1$, and $n_{l}<0$.

After estimating the time-domain variation of all paths over an OFDM symbol period, the estimated channel matrix in time-domain $\left(\hat{\mathscr{H}}^{*}\right)$ can be composed as follows (for simplicity, the OFDM symbol index $i$ is omitted):

$$
\widehat{\mathscr{H}}^{*}=\left[\begin{array}{c}
\widehat{\mathbf{h}}_{0} \\
\widehat{\mathbf{h}}_{1} \\
\vdots \\
\widehat{\mathbf{h}}_{L-1}
\end{array}\right]^{T}=\left[\begin{array}{cccc}
\widehat{h}_{0,0} & \widehat{h}_{0,1} & \ldots & \widehat{h}_{0, N-1} \\
\widehat{h}_{1,0} & \widehat{h}_{1,1} & \ldots & \widehat{h}_{1, N-1} \\
\vdots & \vdots & \vdots & \vdots \\
\widehat{h}_{L-1,0} & \widehat{h}_{L-1,1} & \ldots & \widehat{h}_{L-1, N-1}
\end{array}\right] \text {, }
$$

where $\hat{\mathscr{H}}^{*} \in \mathbb{C}^{L \times N}$.

Combining (10) with (11), we can define the new linear interpolation model in a matrix form as

$$
\widehat{\mathscr{H}}^{*}=\frac{1}{N_{T}-1} \operatorname{diag}\left(\boldsymbol{\Psi}\left[\mathbf{r}^{(i+1)}-\mathbf{r}^{(i)}\right]\right) \mathbf{M}+\Psi_{\mathbf{r}^{(i)}} \mathbf{J}_{1 \times N},
$$

in which

$$
\mathbf{M}=\mathbf{J}_{L \times 1} \mathbf{V}-\mathbf{W J}_{1 \times N} \in \mathbb{C}^{L \times N},
$$

where

$$
\begin{aligned}
\mathbf{V} & =[0,1,2, \ldots, N-1] \in \mathbb{C}^{1 \times N} \\
\mathbf{W} & =\left[n_{0}, n_{1}, n_{2}, \ldots, n_{L-1}\right]^{T} \in \mathbb{C}^{L \times 1}
\end{aligned}
$$

In order to obtain the OFDM channel matrix, $\hat{\mathscr{H}}$, we rearrange the entries of $\widehat{\mathscr{H}}^{*}$ as follows:

$$
\hat{\mathscr{H}}=\left[\begin{array}{ccccccc}
\widehat{h}_{0,0} & 0 & \ldots & 0 & \widehat{h}_{L-1,0} & \ldots & \widehat{h}_{1,0} \\
\widehat{h}_{1,1} & \widehat{h}_{0,1} & 0 & \ddots & 0 & \ddots & \widehat{h}_{2,0} \\
\vdots & \ddots & \ddots & \ddots & \ddots & \ddots & \vdots \\
\widehat{h}_{L-1, L-1} & \vdots & \ddots & \ddots & \ddots & \ddots & 0 \\
0 & \widehat{h}_{L-1, L} & \ddots & \ddots & \ddots & \ddots & \vdots \\
\vdots & \ddots & \ddots & \ddots & \ddots & \ddots & 0 \\
0 & \ldots & 0 & \widehat{h}_{L-1, N-1} & \ldots & \widehat{h}_{1, N-1} & \widehat{h}_{0, N-1}
\end{array}\right]
$$


Finally, the received symbol, $\mathbf{Y}$, at the output of DFT is equalized (by multiplying the vector $\mathbf{Y}$ by the inverse of estimated frequency-domain channel matrix $\widehat{\mathbf{H}}$ ):

$$
\widehat{\mathbf{X}}=\widehat{\mathbf{H}}^{-1} \mathbf{Y} \text {, }
$$

in which

$$
\widehat{\mathbf{H}}=\mathbf{F} \hat{\mathscr{H}} \mathbf{F}^{H},
$$

where $\mathbf{F}^{H}$ is the IDFT matrix.

5.1. Complexity and Noise Reduction (CNR) Criterion. In most cases, mobile communication channel has only a few dominant channel paths, compared to maximum delay spread (see Table 1 as an example). Hence, many samples of the CIR have little or no energy and comprise noise, removing these nondominant paths will reduce the estimator complexity, and it is expected to improve its performance. Therefore, to remove those nondominant paths and consequently reduce the noise effect, all paths are compared against a threshold.

The optimum threshold requires knowledge of the signal-to-noise ratio, which is not practically feasible for a very high Doppler scenario. Hence, we suggested a simple but effective method to remove most of those paths. The suggested threshold depends on the maximum absolute value of the CIR and also on its mean value as follows:

$$
\text { Threshold }=\frac{\beta \mathbb{E}\left[\widehat{h}_{l, n_{l}}^{(i)}\right]}{\max \left|\widehat{h}_{l, n_{l}}^{(i)}\right|}, \quad 0 \leq \beta \leq 1,0 \leq l \leq L-1,
$$

where the parameter $\beta$ is used to set the threshold. If $\beta$ is set to low, some noise components are treated as channel paths. On the contrary, if $\beta$ is set high, some dominant channel paths are removed.

In our simulation in Section 6 , we set $\beta=0.50$, such that most of the dominant channel paths are preserved. Moreover, to reduce the probability of erroneously zeroing dominant channel paths, the final decision to set the current path to zero is based on both the current and next CIRs as follows:

$$
\text { If both }\left|\widehat{h}_{l, n_{l}}^{(i)}\right| \text { and }\left|\widehat{h}_{l, n_{l}}^{(i+1)}\right| \leq \text { Threshold } \Longrightarrow \widehat{h}_{l, n_{l}}^{(i)}=0 \text {. }
$$

Therefore, by implementing the CNR criterion, instead of estimating numerous channel paths, only few dominant channel paths are estimated. Hence, the CNR criterion substantially reduces the computational complexity. Additionally, we expect better BER performance due to removing those noise perturbation paths.

5.2. Mean-Square Error Analysis. In this subsection, we derive an analytical expression for the mean-squared error (MSE) under some reasonable assumptions. In our scheme, the estimation errors come from linear interpolation and AWGN components in the received pilot samples. First, we assume the channel varies linearly within the OFDM symbol
TABLE 1: OFDM simulation parameters.

\begin{tabular}{lc}
\hline Parameters & Specifications \\
\hline IFFT, FFT size $(N)$ & 1024 \\
Subcarrier spacing $(\Delta f)$ & $10.94 \mathrm{kHz}$ \\
Signal constellation & 16 QAM, 64 QAM \\
Effective symbol duration $\left(T_{u}\right)$ & $91.40 \mu \mathrm{s}$ \\
Guard interval $\left(T_{g}\right)$ & $5.71 \mu \mathrm{s}$ \\
Sampling interval $\left(T_{s}\right)$ & $89.26 \mathrm{~ns}$ \\
Bandwidth & $11.20 \mathrm{MHz}$ \\
Number of OFDM symbols $(I)$ & 3000 \\
Carrier frequency $\left(f_{c}\right)$ & $3 \mathrm{GHz}$ \\
\hline
\end{tabular}

and is noiseless. Hence, in this case, the proposed scheme will estimate the channel perfectly without any error.

5.2.1. Noise-Free Case. Let the true value of the channel path at instant time $n$ be $h_{l, n}^{(i)}$. Then, by using LS estimation, the estimated CIR at pilot samples for the $l$ th path, $0 \leq l \leq L-1$, can be found as follows:

$$
\begin{gathered}
\hat{h}_{l, n_{l}}^{(i)}=\frac{A h_{l, n_{l}}^{(i)}}{A}=h_{l, n_{l}}^{(i)}, \\
\widehat{h}_{l, n_{l}}^{(i+1)}=\frac{A h_{l, n_{l}}^{(i+1)}}{A}=h_{l, n_{l}}^{(i+1)} .
\end{gathered}
$$

Substituting, $\widehat{h}_{l, n_{l}}^{(i)}$ and $\widehat{h}_{l, n_{l}}^{(i+1)}$ into (10), we have

$h_{l, n}^{(i)}=\frac{\left(h_{l, n_{l}}^{(i+1)}-h_{l, n_{l}}^{(i)}\right)\left(n-n_{l}^{(i)}\right)}{N_{T}-1}+h_{l, n_{l}}^{(i)}, \quad 0 \leq n \leq N-1$.

Equation (20) provides an error free estimate of the $l$ th channel path.

5.2.2. Noise Case. In this case, we assume the channel varies linearly over an OFDM symbol and take into account the noise effect. Then, by using LS estimation, the estimated CIR at pilot samples can be found as follows:

$$
\begin{gathered}
\widehat{h}_{l, n_{l}}^{(i)}=h_{l, n_{l}}^{(i)}+\frac{w_{l, n_{l}}^{(i)}}{A}, \\
\widehat{h}_{l, n_{l}}^{(i+1)}=h_{l, n_{l}}^{(i+1)}+\frac{w_{l, n_{l}}^{(i+1)}}{A} .
\end{gathered}
$$

Substituting $\widehat{h}_{l, n_{l}}^{(i)}$ and $\widehat{h}_{l, n_{l}}^{(i+1)}$ into (10) yields, after simple math manipulation,

$$
\begin{aligned}
\widehat{h}_{l, n}^{(i)}= & \frac{\left(h_{l, n_{l}}^{(i+1)}-h_{l, n_{l}}^{(i)}\right)\left(n-n_{l}^{(i)}\right)}{N_{T}-1}+h_{l, n_{l}}^{(i)} \quad 0 \leq n \leq N-1 . \\
& +\frac{\left(w_{l, n_{l}}^{(i+1)}-w_{l, n_{l}}^{(i)}\right)\left(n-n_{l}^{(i)}\right)}{A\left(N_{T}-1\right)}+\frac{w_{l, n_{l}}^{(i)}}{A}
\end{aligned}
$$

Considering (20), in the noise-free case, (22) can be rewritten in terms of $h_{l, n}^{(i)}$ as 


$$
\hat{h}_{l, n}^{(i)}=h_{l, n}^{(i)}+\frac{\left(w_{l, n_{l}}^{(i+1)}-w_{l, n_{l}}^{(i)}\right)\left(n-n_{l}^{(i)}\right)}{A\left(N_{T}-1\right)}+\frac{w_{l, n_{l}}^{(i)}}{A} .
$$

The MSE of the $l$ th channel path during the $i$ th OFDM symbol interval can be defined as

$$
\mathrm{MSE}=\mathbb{E}\left[\left|\widehat{h}_{l, n}^{(i)}-h_{l, n}^{(i)}\right|^{2}\right], \quad 0 \leq n \leq N-1 .
$$

Substituting (23) into (24), after some manipulation, we have

$$
\begin{aligned}
M S E= & \mathbb{E}\left\{\left[\frac{\left(w_{l, n_{l}}^{(i+1)}\right)^{2}-2 w_{l, n_{l}}^{(i+1)} w_{l, n_{l}}^{(i)}+\left(w_{l, n_{l}}^{(i)}\right)^{2}}{A^{2}\left(N_{T}-1\right)^{2}}\right]\left(n-n_{l}^{(i)}\right)^{2}\right\} \\
& +\mathbb{E}\left\{\left[\frac{\left.\left.2 w_{l, n_{l}}^{(i+1)} w_{l, n_{l}}^{(i)}-\frac{2\left(w_{l, n_{l}}^{(i)}\right)^{2}}{A^{2}\left(N_{T}-1\right)}\right]\left(n-n_{l}^{(i)}\right)\right\}}{A^{2}\left(N_{T}-1\right)}\right]\right. \\
& +\mathbb{E}\left[\frac{\left(w_{l, n_{l}}^{(i)}\right)^{2}}{A^{2}}\right] .
\end{aligned}
$$

Since the noise is modeled as complex AWGN with zero mean and unit variance, we can rewrite (25) as

$$
\text { MSE }=\frac{\sigma_{w_{l, n_{l}}}^{2}}{A^{2}}\left\{2 \mathbb{E}\left[\frac{\left(n-n_{l}^{(i)}\right)^{2}}{\left(N_{T}-1\right)^{2}}\right]-2 \mathbb{E}\left[\frac{n-n_{l}^{(i)}}{N_{T}-1}\right]+1\right\} .
$$

Then, we have the following approximations (derived in Appendixes A and B):

$$
\begin{aligned}
\mathbb{E}\left[\frac{\left(n-n_{l}^{(i)}\right)^{2}}{\left(N_{T}-1\right)^{2}}\right] & \approx \frac{1}{3}, \\
\mathbb{E}\left[\frac{n-n_{l}^{(i)}}{N_{T}-1}\right] & \approx \frac{1}{2} .
\end{aligned}
$$

Thus, we have

$$
\operatorname{MSE} \approx \frac{2}{3 A^{2}} \sigma_{w_{l, n_{l}}}^{2} .
$$

Since the channel paths are statistically independent. Therefore, a general formula for the MSE of the proposed estimator with $L$ channel paths can be written as follows:

$$
\text { MSE } \approx \frac{2 L}{3 A^{2}} \sigma_{w}^{2} \text {. }
$$

5.3. Complexity Comparison. Here, we briefly discuss the main complexity of the proposed estimator in terms of the required complex multiplications and additions/subtractions operations. In the proposed scheme, the channel path is obtained directly by extracting the CIR from the timedomain received signal. The complexity of our scheme mainly exists in the linear interpolation between CIRs.
According to (12) and (13), our analysis shows that the computational complexity of calculating $M$ and $\widehat{\mathscr{H}}^{*}$ are $\mathcal{O}\left(3 L_{M D P} N\right)$ and $\mathcal{O}\left(L_{M D P}^{2} N+3 L_{M D P} N+N_{T}+L_{M D P} N_{T}\right)$ $\approx \mathcal{O}\left(L_{M D P}^{2} N+4 L_{M D P} N\right)$, respectively. Therefore, the overall complexity of the proposed scheme is approximately equal to $\mathcal{O}\left(L_{M D P}^{2} N+7 L_{M D P} N\right)$, where $L_{M D P}$ is the number of most dominant channel paths $\left(L_{M D P} \ll N_{g}\right)$.

On the contrary, the complexity of the BSBL scheme, which is used for comparison, is given by $\mathcal{O}\left(Q^{3} L^{2} N_{\text {iter }}+\right.$ $Q^{3} N_{g}^{3} N_{\text {iter }}$ ) [19]. In addition to the direct complexity of BSBL scheme, the number of required iterations $\left(N_{i t e r}\right)$ for convergence is another issue for complexity analysis. Furthermore, simulations (Section 6) show that the BSBL scheme can work with a significant large $N_{i t e r}$. However, the complexity of the BSBL estimator is usually too high for practical use.

Finally, both schemes need a matrix inversion to perform the equalization in the frequency domain, in which computational complexity is high, especially for large OFDM symbol sizes $(N)$. It should be emphasized here that our scheme achieves better performance while providing dramatically lower complexity.

\section{Simulation Results}

To investigate the performance of our estimator, a comparison is made between the BER performance of OFDM with our scheme and BSBL scheme [19], which uses the pilot subcarriers to estimate the time-domain channel matrix. Additionally, a BER performance of OFDM with perfect channel state information (CSI) is considered. Furthermore, the performance of channel estimators is evaluated by using the normalized mean square error (NMSE), which is defined as

$$
\mathrm{NMSE}=\frac{1}{I} \sum_{i=1}^{I} \frac{\left\|\widehat{\mathbf{H}}^{(i)}-\mathbf{H}^{(i)}\right\|_{F}^{2}}{\left\|\mathbf{H}^{(i)}\right\|_{F}^{2}},
$$

where $I$ is the number of the channel realizations and $\widehat{\mathbf{H}}^{(i)}$ is the estimated channel matrix of $\mathbf{H}^{(i)}$ at the ith realization.

In the simulation, an uncoded OFDM system with 16quadrature amplitude modulation (QAM) is considered. Moreover, the new scheme is also tested with the higherorder modulation constellations of 64-QAM. The relevant OFDM system parameters are given in Table 1.

To verify the performance of the proposed scheme, two different channel models are considered. The power-delay profile (PDP) of channel \#1 is the COST 207 typical urban six-path (TU6) model which is given in Table 2. However, the PDP of channel \#2 has two paths with power profile [0.64 0.34 ], which are separated by $5.7 \mu \mathrm{s}$. In addition, each channel path weight is simulated based on Jakes' Rayleigh model [22].Also, two maximum normalized Doppler spreads of $\varepsilon=0.02$ and 0.07 are considered, corresponding to a mobile terminal moving at maximum speeds of 78 and $276 \mathrm{~km} / \mathrm{h}$, respectively. Moreover, based on the simulation results, the amplitude of the pilot signal is set equal to 10 , which gives the best results for our scheme. Furthermore, in 
TABle 2: Power-delay profile of channel \#1.

\begin{tabular}{lcccccc}
\hline Path index & 1 & 2 & 3 & 4 & 5 & 6 \\
\hline Delay $(\mu \mathrm{s})$ & 0.0 & 0.2 & 0.5 & 1.6 & 2.3 & 5.0 \\
Average power $(\mathrm{dB})$ & -3 & 0 & -2 & -6 & -8 & -10 \\
\hline
\end{tabular}

the new scheme, the OFDM is zero-padded by $2 N_{g}+1$ samples; thus, the spectral efficiency is reduced. Hence, for fair comparison, 47 equally spaced subcarriers are allocated for pilot subcarriers in the BSBL scheme. Therefore, both schemes have approximately the same spectral efficiency. Moreover, the same transmitted power is used for both schemes.

Figure 3 shows a comparison of the analytical and simulation results of MSE for the proposed scheme. At high $E_{b} / N_{0} s(>20 \mathrm{~dB})$, excellent agreement between the analytical and simulation results is observed. However, at low $E_{b} / N_{0} s(<20 \mathrm{~dB})$, the difference between the analytical and simulation results decreases with increasing $E_{b} / N_{0} s$. This can be explained as follows; according to (29), the MSE is directly proportional to the number of paths. At high $E_{b} / N_{0} s$, the noise power is low. Hence, the CNR criterion removes most of the nondominant paths (noise perturbation paths), so the number of estimated paths is approximately equal to the actual number of channel paths. Hence, there is great agreement between the theoretical and simulation MSE.

On the contrary, at low $E_{b} / N_{0} s$, the noise power is high. Hence, the CNR criterion removes few nondominant paths, so the number of estimated channel paths is very large compared with the actual channel paths. Hence, theoretical and simulation MSE results are not identical.

Figure 4 shows BER vs. $E_{b} / N_{0}$ for 16QAM-OFDM with the proposed and BSBL schemes over channel \#1, for different values of normalized Doppler frequency $(\varepsilon=0.02$ and $\varepsilon=0.07)$. In case of $\varepsilon=0.01$, at low $E_{b} / N_{0} s(<25 \mathrm{~dB})$, it is clear that our scheme consistently outperforms BSBL scheme whereas, at high $E_{b} / N_{0} s(>25 \mathrm{~dB})$, our scheme significantly outperforms the BSBL scheme. This is due to the fact that, at low $E_{b} / N_{0} s$, the noise significantly affects the amplitude of the pilot sample, which severely degrades the performance of the proposed scheme. Furthermore, in case of $\varepsilon=0.07$, one can observe that our scheme effectively reduces the error floor. Moreover, in both cases, $\varepsilon=0.02$ and $\varepsilon=0.07$, the performance of the proposed scheme is very close to the performance of CSI. Also, one can observe, in case of $\varepsilon=0.02$, our scheme has a slight performance gain over CSI. This is not a surprising result, since LS estimate yields some noise reduction.

Figure 5 shows a BER performance comparison for 64QAM-OFDM, over channel \#1. In case $\varepsilon=0.07$, one can observe that, at low $E_{b} / N_{0}(<10 \mathrm{~dB})$, the proposed scheme can only achieve a slight improvement in BER performance over the BSBL scheme. However, at high $E_{b} / N_{0} s$, our scheme can achieve impressive BER performance compared with BSBL scheme, particularly, when $E_{b} / N_{0} s>20 \mathrm{~dB}$.

Additionally, from Figure 5, in case of $\varepsilon=0.02$, the performance of our scheme shows a very good agreement

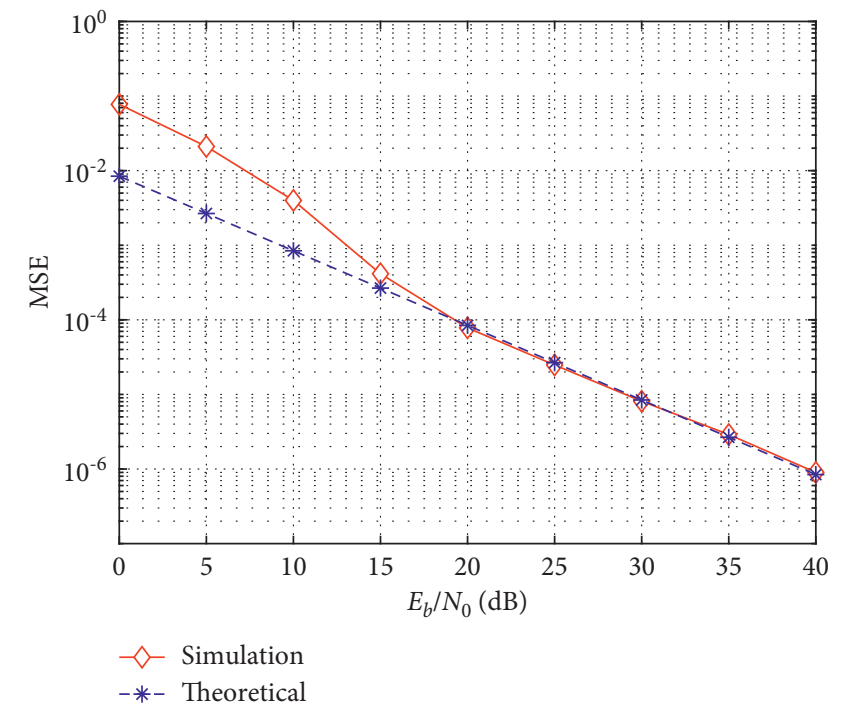

FIgURE 3: The MSE of the proposed estimator for 16QAM-OFDM over channel \#1, with vehicle moving at $40 \mathrm{~km} / \mathrm{h}$.

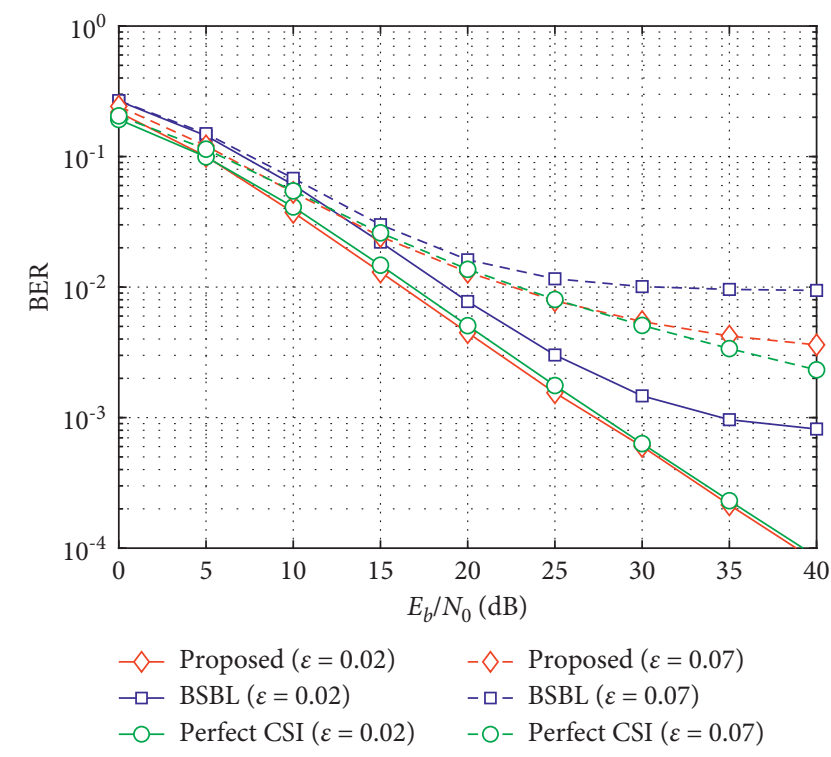

FIGURE 4: BER performance comparison among different channel estimators for 16QAM-OFDM over channel \#1.

with the CSI. However, in case of $\varepsilon=0.07$, the proposed scheme and CSI have different performance when $E_{b} / N_{0} s>30 \mathrm{~dB}$. A clear improvement in BER performance of the proposed scheme can be observed compared with the BSBL scheme. More specifically, the effect of ICI resulting in an error floor is significantly decreased. This can be explained by the fact that, in pilot subcarrier-based estimator (for example, BSBL scheme), the pilot itself suffers from ICI. Consequently, the channel estimator accuracy degrades as ICI increases. However, in pilot sample-based estimator (for example, the proposed scheme), the pilot is affected only by AWGN. Also, one can see from Figure 5, in case of $\varepsilon=0.02$, that the proposed scheme consistently outperforms BSBL scheme, and it can achieve a BER of $1.1 \times 10^{-3}$ at 


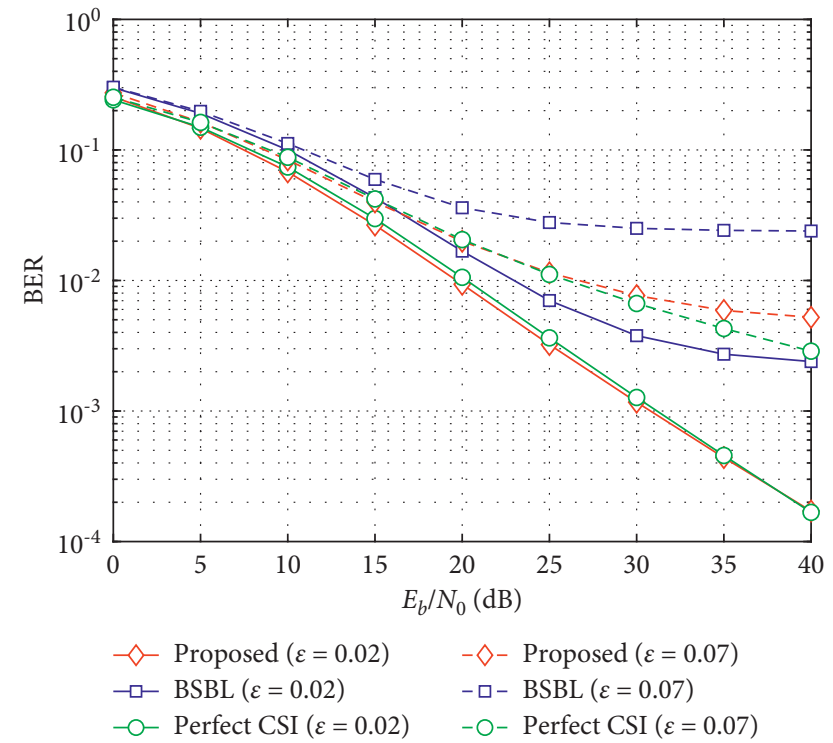

FIGURE 5: BER performance comparison among different channel estimators for 64-QAM-OFDM over channel \#1.

$E_{b} / N_{0}=30 \mathrm{~dB}$. At a BER of $2 \times 10^{-3}$, our estimator outperforms the BSBL scheme by about $3 \mathrm{~dB}$.

A BER performance comparison for 16QAM-OFDM with the proposed and BSBL schemes over channel \#2 is shown in Figure 6. In case $\varepsilon=0.02$, one can observe that our scheme outperforms the BSBL scheme. However, $\varepsilon=0.07$, and our scheme significantly outperforms the BSBL scheme, particularly, at high $E_{b} / N_{0} s$. This can be explained by noting that the channel has only two dominant paths, so the proposed scheme with CNR criterion removes many noise perturbation paths, which results in significant noise reduction. As a consequence, substantial performance improvement is compared with Figure 4. On the contrary, the BSBL scheme shows slight performance improvement compared with Figure 4.

Figure 7 illustrates a BER performance comparison as a function of $\varepsilon \%$ for 16QAM-OFDM. In these comparisons, channel \#1 is used and $E_{b} / N_{0}$ is set to $30 \mathrm{~dB}$. In the same figure, one can see that the proposed scheme significantly outperforms the BSBL scheme in BER performance, particularly, when $\varepsilon<10 \%$. Moreover, the BER performance of our estimator is very close to that with perfect channel knowledge, when $\varepsilon<7 \%$. However, when $\varepsilon<7 \%$, the gap between our estimator and CSI gradually increases with respect to $\varepsilon$. This can be explained that the channel variation within OFDM symbols is no longer linear, whereas the proposed scheme uses a linear model to approximate the channel variation.

Figure 8 shows NMSE comparison of the estimated frequency-domain channel for 16QAM-OFDM with the proposed and BSBL schemes over channel \#1. When $\varepsilon=0.02$, our scheme estimates the channel perfectly; however, when $\varepsilon=0.07$, the proposed scheme fails to track channel variations within OFDM symbols, which is not surprising, since, in this case, the channel variation no longer linear. Hence, the linear approximation becomes ineffective,

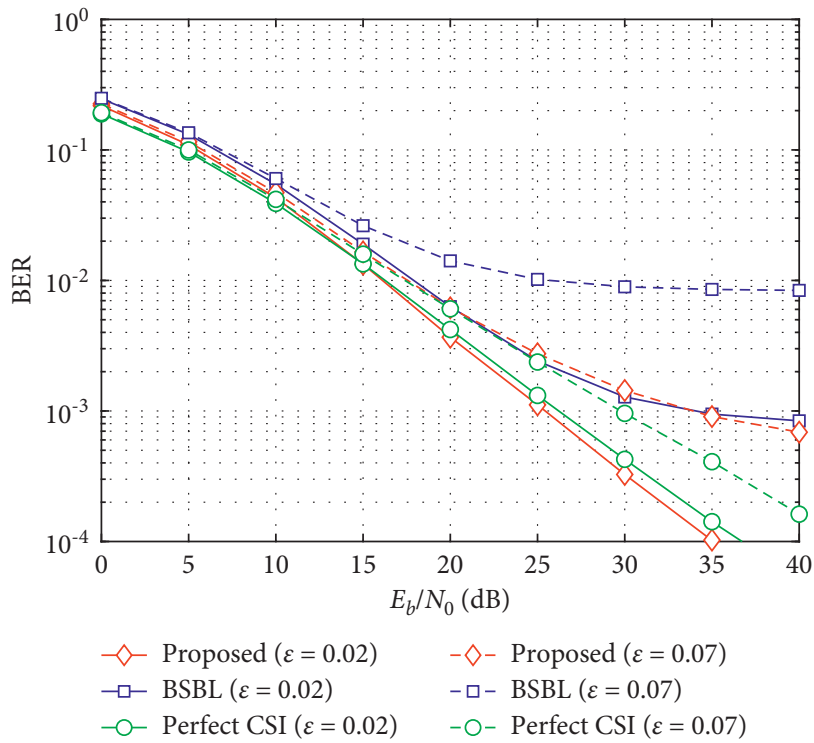

FIGURE 6: BER performance comparison among different channel estimators for 16QAM-OFDM over channel \#2.

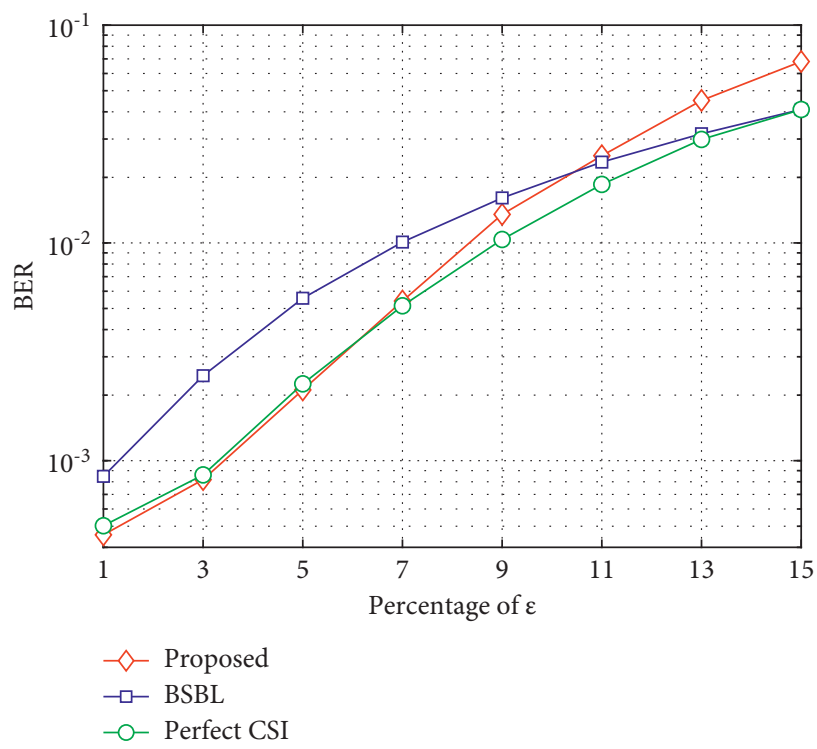

FIGURE 7: BER performance comparison as a function of $\varepsilon$ among different channel estimators for 16QAM-OFDM over channel \#1, with $E_{b} / N_{0}=30 \mathrm{~dB}$.

in this case, and leads to an error floor which cannot be overcome by increasing $E_{b} / N_{0}$.

Figure 9 illustrates the BER performance for 16QAMOFDM with the two estimation schemes versus $E_{b} / N_{0}$ and the maximum velocity over channel \#1. It can be seen that the rate of the BER scheme increases as velocity increases for fixed values of $E_{b} / N_{0}$. Also, in Figure 9, one can see that the proposed scheme can significantly improve the BER performance as compared to the BSBL scheme in the high $E_{b} / N_{0}$ and low-speed regions, especially when $E_{b} / N_{0}>20 \mathrm{~dB}$ and the velocity $<400 \mathrm{~km} / \mathrm{h}$. However, in the 


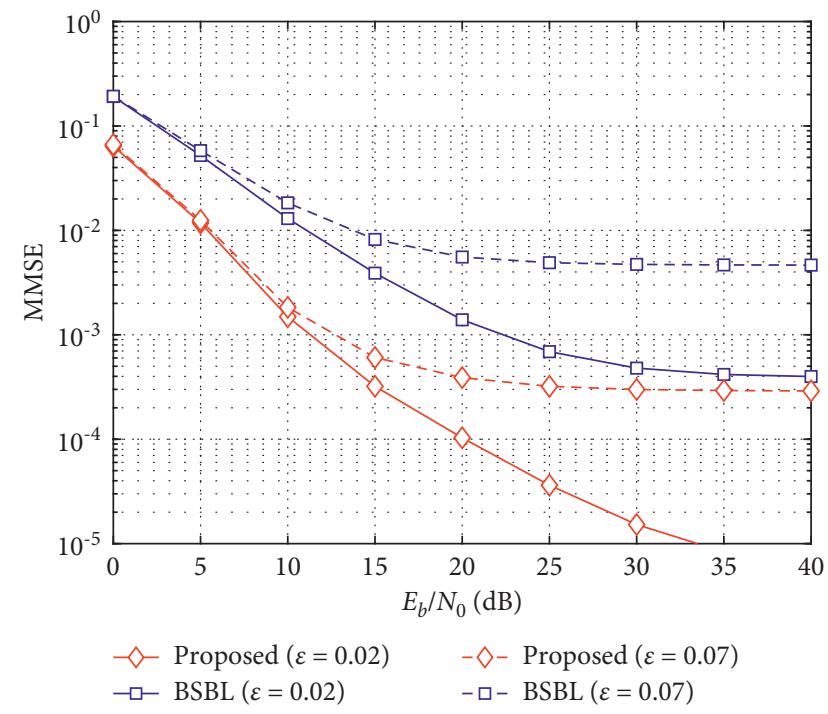

Figure 8: Mean square channel estimation error between the proposed and BSBL for 16QAM-OFDM over channel \#1.

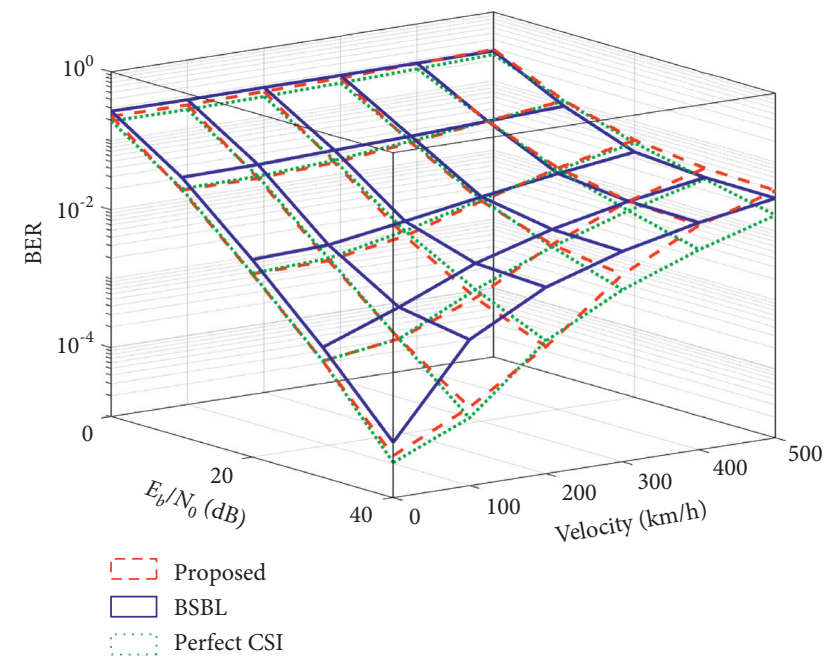

FIGURE 9: BER performance comparison among different channel estimators for 16QAM-OFDM over channel \#1, at different speeds.

high-speed regions, both schemes suffer large estimation errors, which are due to the fact that, in high mobility, the channel paths show high-order time variation, whereas pilot subcarriers strongly suffer from ICI.

\section{Conclusions}

In this paper, a new scheme, which is mainly based on a sample pilot with ZP in the time domain, is proposed to estimate the channel variation over an OFDM symbol period. Simulation results of MSE, showing excellent agreement with theoretical analysis, confirm the validity of our MSE analysis. Also, simulation results demonstrate that the proposed scheme can greatly improve the BER performance of the OFDM system as compared with the BSBL scheme, in particular, at high $E_{b} / N_{0} s$. Additionally, results show that the error floor resulting from the effect of ICI is significantly decreased by implementing the new scheme. Furthermore, the BER performance of our scheme can nearly achieve the performance as good as the perfect knowledge of channel state information when $\varepsilon<0.02$. It is expected that our estimator will achieve best results when both the OFDM symbol duration and the channel delay spread are small, since, in this case, the separation between any two adjacent pilot signals is minimal. The complexity of the new estimator is significantly lower than BSBL, while showing a better performance.

\section{Appendix}

\section{A. Analysis of the First Term in (20)}

$$
\begin{aligned}
& \mathbb{E}\left[\left(n-n_{l}^{(i)}\right)^{2}\right]=\frac{1}{N} \sum_{n=0}^{N-1}\left(n-n_{l}^{(i)}\right)^{2}, \\
& \mathbb{E}\left[\left(n-n_{l}^{(i)}\right)^{2}\right]=\frac{2 N^{2}-3 N+1-6 n_{l}^{(i)} N+6 n_{l}^{(i)}+6 n_{l}^{(i)^{2}}}{6} .
\end{aligned}
$$

Then, we have the approximation:

$$
\begin{aligned}
& \mathbb{E}\left[\left(n-n_{l}^{(i)}\right)^{2}\right] \approx \frac{2 N^{2}-6 n_{l}^{(i)} N+6 n_{l}^{(i)^{2}}}{6}, \\
& \mathbb{E}\left[\frac{\left(n-n_{l}^{(i)}\right)^{2}}{\left(N_{T}-1\right)^{2}}\right] \approx \frac{2 N^{2}-6 n_{l}^{(i)} N+6 n_{l}^{(i)^{2}}}{6\left(N_{T}-1\right)^{2}} .
\end{aligned}
$$

For large symbol duration and small delay spread, we obtain the approximation:

$$
\mathbb{E}\left[\frac{\left(n-n_{l}^{(i)}\right)^{2}}{\left(N_{T}-1\right)^{2}}\right] \approx \frac{1}{3} .
$$

\section{B. Analysis of the Second Term in (20)}

$$
\begin{aligned}
& \mathbb{E}\left[n-n_{l}^{(i)}\right]=\frac{1}{N} \sum_{n=0}^{N-1}\left(n-n_{l}^{(i)}\right), \\
& \mathbb{E}\left[\frac{n-n_{l}^{(i)}}{N_{T}-1}\right]=\frac{N-2 n_{l}^{(i)}-1}{2\left(N_{T}-1\right)} .
\end{aligned}
$$

For large symbol duration and small delay spread, we have the approximation:

$$
\mathbb{E}\left[\frac{n-n_{l}^{(i)}}{N_{T}-1}\right] \approx \frac{1}{2} .
$$

\section{Data Availability}

No data were used to support this study. 


\section{Conflicts of Interest}

The authors declare that they have no conflicts of interest.

\section{References}

[1] Z. Sheng, H. D. Tuan, H. H. Nguyen, and Y. Fang, "Pilot optimization for estimation of high-mobility OFDM channels," IEEE Transactions on Vehicular Technology, vol. 66, no. 10, pp. 8795-8806, 2017.

[2] Y. P. Wibawa, Iskandar, and I. Zakia, "Filtered-OFDM for high-speed railway communications," in Proceedings of the 2020 6th International Conference on Wireless and Telematics (ICWT' 2020), pp. 1-5, Yogyakarta, Indonesia, September 2020.

[3] G. Noh, B. Hui, and I. Kim, "High speed train communications in 5G: design elements to mitigate the impact of very high mobility," IEEE Wireless Communications, vol. 27, no. 6, pp. 98-106, 2020.

[4] Z. Ma, Z. Zhang, Z. Ding, P. Fan, and H. Li, "Key techniques for $5 \mathrm{G}$ wireless communications: network architecture, physical layer, and MAC layer perspectives," Science China Information Sciences, vol. 58, no. 4, pp. 1-20, 2015.

[5] T. Nakamura, "Requirements for further advancements for evolved universal terrestrial radio access (E-UTRA) (LTEadvanced) (release 14)," Techrep Marketing, vol. 36, p. 913, 2014.

[6] X. Ma, F. Yang, S. Liu, J. Song, and Z. Han, "Sparse channel estimation for MIMO-OFDM systems in high-mobility situations," IEEE Transactions on Vehicular Technology, vol. 67, no. 7, pp. 6113-6124, J2018.

[7] Z. A. H. Qasem, H. A. Leftah, H. Sun, J. Qi, and H. Esmaiel, "X-transform time-domain synchronous IM-OFDM-SS for underwater acoustic communication," IEEE Systems Journal, pp. 1-12, 2021.

[8] Z. A. H. Qasem, J. Wang, X. Kuai, H. Sun, and H. Esmaiel, "Enabling unique word OFDM for underwater acoustic communication," IEEE Wireless Communications Letters, vol. 10, no. 9, pp. 1886-1889, 2021.

[9] H. Senol, "Joint channel estimation and symbol detection for OFDM systems in rapidly time-varying sparse multipath channels," Wireless Personal Communications, vol. 82, no. 3, pp. 1161-1178, 2015.

[10] L. Liang Zhang, Z. Zhihong Hong, L. Thibault, R. Boudreau, and $\mathrm{Y}$. Yiyan $\mathrm{Wu}$, "A low-complexity robust OFDM receiver for fast fading channels," IEEE Transactions on Broadcasting, vol. 60, no. 2, pp. 347-357, 2014.

[11] Y. Peng, W. Wang, and Y. il Kim, "Performance analysis of OFDM system over time-selective fading channels," in Proceedings of the 2009 IEEE Wireless Communications and Networking Conference (WCNC' 2009), pp. 1-5, Budapest, Hungary, April 2009.

[12] H.-C. Wu, "Analysis and characterization of intercarrier and interblock interferences for wireless mobile OFDM systems," IEEE Transactions on Broadcasting, vol. 52, no. 2, pp. 203-210, J2006.

[13] Q. Tang, H. Long, H. Yang, and Y. Li, "An enhanced LMMSE channel estimation under high speed railway scenarios," in Proceedings of the 2017 IEEE International Conference on Communications Workshops (ICC Workshops), pp. 999-1004, Paris, France, May 2017.

[14] M. Almoneer, C. Rohde, K. Hassan, and W. H. Gerstacker, "Intercarrier interference-aware pilot-aided channel estimation in OFDM systems," IEEE Transactions on Broadcasting, vol. 63, no. 3, pp. 449-462, 2017.

[15] P. Chen and H. Kobayashi, "Maximum likelihood channel estimation and signal detection for OFDM systems," in Proceedings of the 2002 IEEE International Conference on Communications, vol. 3, pp. 1640-1645, New York, NY, USA, April 2002.

[16] Y.-H. Yeh and S.-G. Chen, "Reduction of Doppler-induced ICI by interference prediction," in Proceedings of the 2004 IEEE 15th International Symposium on Personal, Indoor and Mobile Radio Communications (IEEE Cat. No.04TH8754), vol. 1, pp. 653-657, Helsinki, Finland, September 2004.

[17] A. Baouni and A. Farrouki, "Channel estimation using a reduced rate of pilot subcarriers for OFDM systems over doubly-selective channels," IET Communications, vol. 12, no. 14, pp. 1714-1721, 2018.

[18] A. N. Uwaechia and N. M. Mahyuddin, "Spectrum-efficient distributed compressed sensing based channel estimation for OFDM systems over doubly selective channels," IEEE Access, vol. 7, pp. 35 072-135088, 2019.

[19] X. Wang, J. Wang, L. He, and J. Song, "Doubly selective underwater acoustic channel estimation with basis expansion model," in Proceedings of the 2017 IEEE International Conference on Communications (ICC' 2017), pp. 1-6, Paris, France, May 2017.

[20] O. Barbu, C. Navarro Manchón, C. Rom, T. Balercia, and B. H. Fleury, "OFDM receiver for fast time-varying channels using block-sparse bayesian learning," IEEE Transactions on Vehicular Technology, vol. 65, no. 12, pp. 10 053-10 057, 2016.

[21] S. S. Tarbosh, T. A. Rahman, and N. Seman, "General pilotaided scheme for OFDM reception in fast varying channels," in Proceedings of the 2012 International Conference on Computer and Communication Engineering (ICCCE), pp. 932-935, Kuala Lumpur, Malaysia, July 2012.

[22] M. F. Pop and N. C. Beaulieu, "Limitations of sum-of-sinusoids fading channel simulators," IEEE Transactions on Communications, vol. 49, no. 4, pp. 699-708, 2001. 